the three partition fractions and their first derivatives with respect to time. The solutions of this system are certainly aperiodic and by the way, the two decay constants become so large, that steady state is asymptotically arrived at in minute fractions, e. $g$. $10^{-8}$, of a second.

Thus periodic variations should be excluded. However, from nerve physiology it is known with certainty that periodic synchronous phenomena actually occur in certain nerves or rather in their end-organs. As now living organisms have so far as known only chemical reactions as ultimate causes of all observable events which take place in them, we may state with a probability approaching certainty that periodic chemical changes actually occur in nature.

To solve this dilemma one might look for mechanisms of the Volterra ${ }^{1}$ type, which allow the appearance of periodic changes. This has been done, i. a. by Bonhoeffer ${ }^{2}$ and Prigogine ${ }^{3,4}$, but such mechanisms must be so artificial that it is hardly possible to believe in their occurrence, e. $g$. in living organisms.

We are therefore turned back on mechanisms of the type described above. Now in solving the corresponding equations we have implicitly assumed, that concentrations and their derivatives with respect to time can be determined simultaneously so accurately as we please. But this may not be true. We can certainly, e. g. by means of a "flash" method, determine the concentra. tions of the forms of the enzyme accurately, at a certain time. But the determination of the derivatives requires a second observation after the lapse of a certain not too short time interval, during which the concentrations may have varied in some unknown way, the more so as the first "flash" to give an observable effect must have changed the state of the enzyme and may have caused some chemical change. Therefore the more accurately we try to determine the concentrations by making the time interval short, the less accurate be. comes the determination of the rate of change and vice versa.

We thus arrive at the result, that the validity of our system of differential equations can neither be proved nor disproved by direct experiments that is, they are physically unrealistic.

Therefore the occurrence of rhythmic chemical changes in the partition fractions need not surprise us, neither be it surprising that it is actually possible, as shown elsewhere ${ }^{5}$, to calculate a frequency of oscillations with time in the values of these fractions.

We have only to remember that the aperiodic and the oscillatory treatment of the kinetic problem mutually exclude each other although separately both are possible.

1. Volterra, V. Théorie mathématique de la lutte pour la vie. Gauthier-Villars 1931.

2. Bonhoeffer, K. F. Naturu iss. 31 (1943) 270, and later papers.

3. Prigogine, I. and Balescu, R. Ac. Roy. Belg. Bull. Cl. Sc. 41 (1955) 917.

4. Prigogine, I. and Balescu, R. Ac. Roy. Belg. Bull. Cl. Sc. 43 (1956) 256.

5. Christiansen, J. A. Acta Chem. Scand. 11 (1957) 1436.

Received January 3, 1958.

\section{Distillation Head for the Distillation of Small Amounts of Liquids}

A R N B B:SON GROTH

Department of Applied Organic Chemistry, Royal Institute of Technology, Stockholm, Sweden

A distillation head suitable for semi-micro distillation columns has been designed and tested. Above all the author has tried. to attain the following results:

1. As small a volume of liquid as possible that will adhere to the walls of the distillation head and the condenser.

2. Absence of stopcocks and ground glass joints.

3. Adjustable and continuous outlet.

4. Fitness for use in distillations at atmospheric pressure as well as under vacuum.

One of the difficulties encountered in the distillation of small amounts of liquid in a column provided with an ordinary distillation head, is the fact that a major part of the liquid to be distilled will remain in the condenser and on the walls of the tube connecting the condenser to the receiver. Usually, this tube has an internal diameter of $1.5-2 \mathrm{~mm}$. Part of this remaining liquid will be in a state of 
continual "remixing" with the new condensate, and in this way will contribute to a decrease of the efficiency of separation. The other fraction, which under normal conditions moves slowly through the narrow tube connecting the outlet stopcock to the receiver, will cause a variable difference between the boiling points at the condenser and the receiver ends. Moreover, a stopcock is usually stained with lubricating grease, and this will influence the purity of the distillate in the distillation of small amounts of liquids.

Sager ${ }^{1}$, Naragon and Lewis ${ }^{2}$, Gould, Holzman and Nieman ${ }^{8}$ have designed distillation heads for semi-micro distillation columns.

The author has designed a distillation head in which the distillate is collected through a capillary tube (about $1 / 10 \mathrm{~mm}$ internal diameter). The inlet of this capillary lies on a somewhat lower level than that of the outlet. In order to be able to obtain a continuous and adjustable stream of distillate from the distillation head to the receiver, it is necessary to have an appropriate pressure difference between the distillation head and the receiver. The manner in which this is reached is demonstrated in the flow sheet shown in Fig. 1. According to this figure, the pressure difference between the distillation head and the receiver is adjusted by means of stopcock d, i.e. the reflux ratio is regulated. For this purpose, a simple and efficient stopcock may consist of a piece of ordinary vacuum hose which can be squeezed, on a

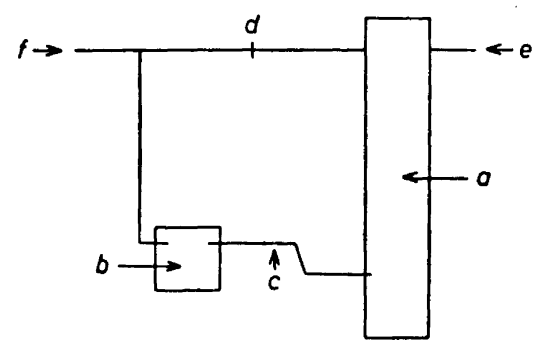

Fig. 1. Flow sheet. a) Distillation head. b) Receiver. c) Capillary tube. d) Adjustable stopcock.

Distillation at atmospheric pressure: e) Introduction of a small gas stream. f) To the surrounding atmosphere.

Distillation under vacuum: e) Introduction of gas through a very fine capillary. f) To vacuum pump.

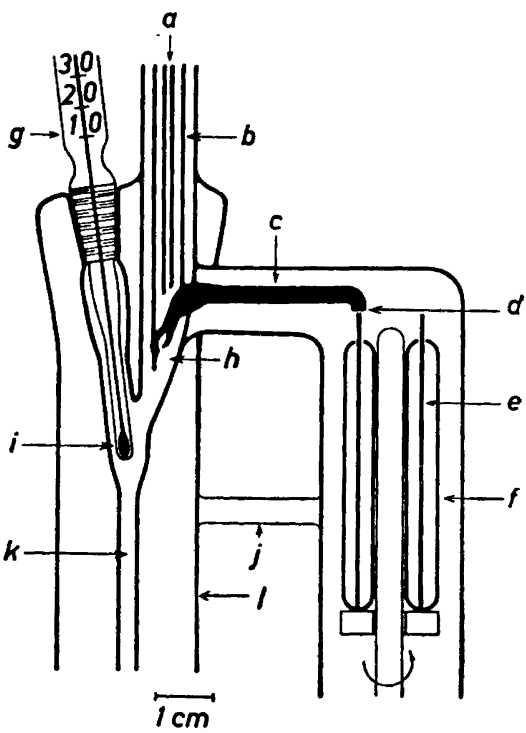

Fig. 2. Description of the distillation head. a) Cooling water. b) Condenser. c) Capillary tube. d) Outlet for distillate. e) Capillary tubes. f) Small test tubes. g) Thermometer. h) Inlet for distillate. i) Thermometer bulb or pocket for a thermocouple (better results are obtained if they are provided with an elongated tip). The distance between the thermometer and the glass walls must be between 1.5 to 2.0 mm. j) Brace. k) Column, $3 \mathrm{~mm}$ internal diameter (the packing of the column is not shown in the figure). 1) Silvered high-vacuum jacket

(the upper part is only halv-silvered).

few centimeters of its length, between two metal plates. This effect can be arrived at by using a clamp for rubber tubes and two elongated (rectangular) metal plates. It is recommended that the diameter of the capillary outlet does not exceed $1 / 10$ $\mathrm{mm}$, since otherwise it will not have enough inertia during the short pressure variations due to an uneven boiling.

The distillation head which has been designed by the author is described in Fig. 2. The distillate is sucked from the drop hanging from the tip of the condenser. It is gathered by means of a system similar to that shown by Gould et al. ${ }^{3}$, i.e. through capillaries standing in small test tubes. The capillaries must then be adjusted so as to reach just under the outlet. 


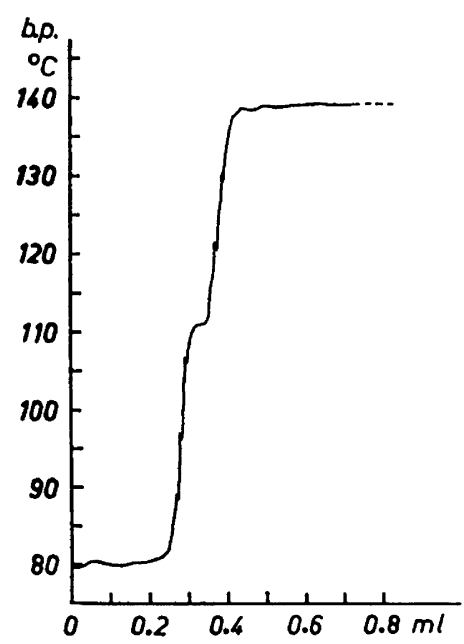

Fig. 3. Distillation curve for a mixture consisting of $0.3 \mathrm{ml}$ benzene, $0.05 \mathrm{ml}$ toluene and $1.0 \mathrm{ml}$ xylene. The temperature was recorded by means of a thermocouple and a recorder.

This will prevent the formation of large drops.

Fig. 3 shows a distillation curve obtained with the apparatus shown in Fig. 2.

It may even be said that this kind of outlet can be applied to larger distillation apparatus. In that case, the system will be designed in such a way that the condensate will drop on the end of the capillary which will have the form of a small cup, with a "dropping tip" at the "outer" bottom.

The construction of the apparatus was executed by the glassblower's firm of Werner Glas (Stockholm).

1. Sager, W. F. in Weissberger, A. Technique of organic chemistry Interscience Publishers, Inc. New York 1951, Vol. IV, p. 486.

2. Naragon, E. A. and Lewis, C. J. Ind. Eng. Chem. Anal. Ed. 18 (1946) 448.

3. Gould, C. W., Holzman, G. and Nieman, C. Ind. Eng. Chem. Anal. Ed. 20 (1948) 361.

Received January 11, 1958.

\section{N-Terminal Amino Acids of Human and Bovine Prothrombin STAFFAN MAGNUSSON}

\author{
Chemistry Department II, Karolinska \\ Institutet, Stockholm 60, Sweden
}

M

ller and van Vunakis ', analyzing bovine prothrombin $(\sim 1500$ NIH $\mathrm{u} / \mathrm{mg}$ ), prepared according to Seegers ${ }^{2}$, found no N-terminal or C-terminal amino acids, using Sanger's 1-fluoro-2,4-dinitrobenzene method and digestion with carboxypeptidase, respectively.

Recently Miller ${ }^{3}$ analyzed bovine prothrombin which he had further purified ( 2000 NIH $\mathrm{u} / \mathrm{mg}$ ) by chromatography on the carboxylic cation exchanger Amberlite IRC 50. He found one mole of N-terminal alanine per mole of prothrombin, again using Sanger's method.

The present author has analyzed bovine and human prothrombin, both prepared according to Seegers ${ }^{2}$, using the phenylisothiocyanate method of Edman for determination of $\mathrm{N}$-terminal amino acids.

Experimental. Bovine prothrombin was prepared from 20 liter portions of bovine blood. The yield of prothrombin, when freeze-drying the isoelectric precipitate at $\mathrm{pH}$ 4.6, after first discarding the precipitate at $\mathrm{pH} 5.4$, was generally $300-400 \mathrm{mg}$.

Human prothrombin was prepared by the same method from one lot of $3200 \mathrm{ml}$ pooled plasma from some fifty donors. The yield was $116 \mathrm{mg}$.

Two methods were used for routine determination of activity.

1. The prothrombin-proconvertin method of Owren and Aas ${ }^{5}$, with the modification that barium sulfate adsorbed bovine plasma according to Brodthagen ${ }^{6}$ was used as source of fibrinogen and factor $V$.

2. Activation of prothrombin by dissolving it in $25 \% \mathrm{w} / \mathrm{w}$ of aqueous trisodium citrate according to Seegers ${ }^{7}$. Clotting activity of the thrombin generated was determined every $15 \mathrm{~min}$, using as substrate bovine fibrinogen (Blombäck and Blombäck ${ }^{8}$ ) in tris(hydroxymethyl)amino methane buffer of ionic strength 0.15 and $\mathrm{pH} 7.5$.

One of our bovine preparations was compared with a sample of prothrombin (No. $55 / 02 / 10$ ) containing $26000 \mathrm{U} / \mathrm{mg}$ tyrosine, obtained from Dr. Seegers and was found to have the same activity as the one of

Acta Chem. Scand. 12 (1958) No. 2 